msh-mss Mathématiques et sciences humaines

162 | Été 2003

Varia

\title{
Ernest Coumet (1933-2003)
}

Ernest Coumet (1933-2003)

Marc Barbut

\section{OpenEdition}

Journals

Édition électronique

URL : http://journals.openedition.org/msh/2887

DOI : $10.4000 / \mathrm{msh} .2887$

ISSN : $1950-682$

\section{Éditeur}

Centre d'analyse et de mathématique sociales de l'EHESS

\section{Édition imprimée}

Date de publication : 1 mars 2003

ISSN : 0987-6936

\section{Référence électronique}

Marc Barbut, « Ernest Coumet (1933-2003) », Mathématiques et sciences humaines [En ligne], 162 | Été 2003, mis en ligne le 10 février 2006, consulté le 23 juillet 2020. URL : http://journals.openedition.org/ $\mathrm{msh} / 2887$ 
ERNEST COUMET (1933 - 2003)

Notre ami Ernest Coumet est mort le 3 janvier 2003. Il y a quelques années, Michel Serfati et moi-même, nous avions souligné dans la préface à un numéro spécial de Mathématiques et Sciences humaines ( ${ }^{\circ} 150,2000$ «La doctrine des chances : sur le calcul des probabilités ») tout ce que la revue et nous-mêmes lui devions. Nous n'étions en cela que les interprètes de la communauté de ceux qui s'intéressent à l'histoire du calcul des probabilités, de la combinatoire et de la logique mathématique.

C'est en effet à ces trois domaines de l'histoire des mathématiques qu'Ernest Coumet a consacré toute une vie de travail. En témoigne la bibliographie très partielle fournie ci-dessous ; en témoignera bientôt l'édition, préparée par le Centre de Synthèse, de ses œuvres complètes, y compris sa thèse sur Mersenne, restée inédite.

Après ses études de licence et un D.E.S. sur « le comportement inductif » (1958) à la Faculté des Lettres et Sciences humaines de Bordeaux, E. Coumet, agrégé de Philosophie, est pensionnaire à la Fondation Thiers et entre au CNRS comme chargé de recherches. Après la soutenance de sa thèse (1968), il est maître-assistant (on ne disait pas encore « maître de conférences ») à l'Université de Paris-X (Nanterre), puis à celle de Paris-I (Panthéon-Sorbonne) dans la section de Philosophie et Épistémologie.

En 1977, il est élu directeur d'études à l'EHESS, où l'accueillera le Centre Alexandre Koyré, mais où se renforceront aussi les liens étroits de collaboration qu'il entretient depuis le début des années 1960 avec le Centre de Mathématique sociale ; les huit articles publiés dans dix numéros de Mathématiques et Sciences humaines, ainsi que d'autres de ses publications (cf. liste ci-dessous) sont le reflet de cette collaboration.

L'École des hautes études en sciences sociales, et surtout la $6^{\mathrm{e}}$ section de l'École pratique des hautes études dont elle est issue, s'est longtemps voulue un établissement marginal par rapport aux autres institutions universitaires ; c'était encore vrai en 1977 lorsqu'Ernest Coumet y est entré. Mais lui-même fut dès lors un «marginal » dans cette École « marginale ».

En effet, comme Pierre Costabel qui fut l'un de ses maîtres, il était d'abord un érudit, et l'érudition avait quelque chose d'incongru dans cette École qui se voulait le temple de la «nouvelle histoire ». Mais son érudition n'empêcha pas, bien au contraire 
elle favorisa le fait qu'il ait toujours pris en compte, en histoire des sciences, l'évolution sur le long terme, chère à $\mathrm{F}$. Braudel.

D'ailleurs, l'histoire des mathématiques telle qu'il la pratiqua et nous l'enseigna, n'opposa jamais histoire sociale et histoire des idées (ou histoire externaliste et histoire internaliste) mais au contraire sut toujours les compléter l'une par l'autre et les appuyer l'une sur l'autre. De cette attitude, son fameux article «La théorie du hasard est-elle née par hasard ?» (Annales E.S.C., 3, 1970) est peut-être l'illustration la plus éclatante.

Cette même érudition associée à la largeur des vues et à la capacité de synthèse se sont retrouvées tout au long de l'existence, depuis deux décennies maintenant, du séminaire d'histoire du calcul des probabilités et de la statistique qu'Ernest Coumet a créé en 1982-83, et dont il fut avec Bernard Bru le principal animateur. Nul n'oubliera ses interventions au séminaire où, ouvrant les célèbres cahiers dans lesquels il préparait chacune des séances, il apportait des compléments et des informations nouvelles au conférencier sur le sujet de celui-ci, et souvent lui en remontrait, toujours avec une bonhommie parfaitement courtoise.

Sa prodigalité, sa générosité dans la transmission de son savoir à chacun de ceux qui ont été ses auditeurs, ses élèves ou ses thésards furent l'un des traits essentiels de cet homme foncièrement bon.

C'est une règle constante que ce sont les épreuves et les souffrances qui révèlent, in fine, le fond du caractère d'un être. Des épreuves, il n'en manqua pas pendant sa jeunesse au Pays Basque et à Bordeaux. Quant aux souffrances, il y eut la perte très tôt de sa jeune femme (1969), et, depuis une dizaine d'années les attaques de plus en plus violentes et cruelles des maladies (car il souffrait de plusieurs maux) qui devaient finalement l'emporter. La constance, le courage et la sérénité avec lesquels il y fit toujours face sont l'ultime preuve de ce qu'il fut, comme on aurait pu le dire lors de son cher XVII ${ }^{\mathrm{e}}$ siècle, à la fois un fort honnête homme et un homme de grande qualité.

M. Barbut

ARTICLES PUBLIÉS DANS Mathématiques et Sciences humaines

[1] «Les jeux de hasard sont-ils une invention du diable ? » $n^{\circ} 6,1964$, p. 23-24.

[2] «Les diagrammes de Venn », $n^{\circ} 10,1965$, p. 31-46.

[3] «À propos de la ruine des joueurs : un texte de Cardan », $\mathrm{n}^{\circ} 11,1965$, p. 19-21.

[4] «Logique, mathématiques et langage dans l'œuvre de George Boole », $\mathrm{n}^{\circ} 15$, 1966, p. 1-14; n 16, 1966, p. 1-14; n 17, 1966, p. 1-12.

[5] «Un texte du XVI ${ }^{\mathrm{e}}$ siècle sur les serrures à combinaisons », $\mathrm{n}^{\circ}$ 22, 1968, p. 33-37.

[6] « Mersenne. Dénombrements, répertoires, numérotations de permutations », $\mathrm{n}^{\circ} 38,1972$, p. 5-37. 
[7] «Sur l'histoire des diagrammes logiques, figures géométriques », n 60, 1977, p. 31-62.

[8] «Histoire du calcul des probabilités et de la statistique », (avec M. Barbut et B. Bru), $\mathrm{n}^{\circ} 113,1991$, p. 57-75.

[9] «Auguste Comte. Le calcul des chances, aberration radicale de l'esprit mathématique », colloque Auguste Comte et l'idée d'une science de l'Homme, Paris, 26-27 novembre 1998, publié dans le présent numéro de Mathématiques et Sciences humaines, p. 9-17.

AUTRES PUBLICATIONS ET COMMUNICATIONS EN RELATION AVEC LE C.A.M.S.

«Réflexions sur les origines du calcul des probabilités. Contrat équitable et règle des partis », Séminaire Modèles mathématiques dans les sciences sociales, Centre de Mathématique Sociale, 4 décembre 1964.

«Leibniz et le problème des partis », Journées Leibniz et la mathématique, Centre Alexandre Koyré et Centre de Mathématique Sociale, 4 mars 1967.

«Des permutations aux XVI ${ }^{\mathrm{e}}$ et XVII ${ }^{\mathrm{e}}$ siècles », in Permutations, Actes du colloque sur les permutations, Paris, 10-13 juillet 1972, Paris, Gauthier-Villars, 1974, p. 277-289.

« Mathématique sociale », entretien avec G.Th. Guilbaud avec la co-participation de P. Ossona de Mendez et P. Rosenstiehl, Paris, AREHESS, coll. Savoir et mémoire $n^{\circ} 4$, 1993, [fascicule et vidéo]. 